\title{
Editorial
}

\section{How much relaxation?}

The dose of muscle relaxants has been largely empirical since their introduction into anaesthesia. The earliest descriptions of the use of "curare" were of modest doses on a background of deep cyclopropane anaesthesia ${ }^{\prime}$ and it was nearly 40 years after the first use of succinylcholine before dose response curves were constructed. ${ }^{2}$ No reversal drugs were given or required. However, as familiarity was gained, the doses increased reaching their zenith in the "Liverpool" technique in which "anaesthesia" was provided, after generous premedication, simply by hyperventilation with nitrous oxide and oxygen using large quantities of curare to maintain relaxation. ${ }^{3}$ Surgical silence was interpreted as acquiescence that an acceptable level of muscle relaxation had been achieved but no attempts were made to determine if optimal conditions had been produced either for surgical relaxation or for restoration of function.

During the last 20 years the careful evaluation of a large number of new neuromuscular blocking compounds with only minor differences in pharmacological activity has encouraged the introduction of a terminology in which the pharmacodynamic behaviour of the drugs can be characterised. For example, Onset Time is defined as the time from administration of a bolus dose (or after the major dose in a priming sequence) until the maximum effect is observed at the adductor pollicis. Clinically, the interval is of little or no importance as it is now recognised that the onset of action of all neuromuscular relaxants is slower at the thumb than at the respiratory ${ }^{4}$ and airway ${ }^{5,6}$ muscles which have greater importance because activity at these sites is likely to be related to intubating conditions. The Recovery Index is the time taken for adductor pollicis twitch activity to recover from 25 to $75 \%$ of control. Whilst this index does provide a value which is useful in comparing the spontaneous rate of recovery of different relaxants, it is of only small clinical importance. Intuitively, one might expect that the more rapid the rate of spontaneous recovery, the less the potential for residual postoperative paralysis and, indeed, this has been demonstrated at several institutions, ${ }^{7-9}$ at least in adult patients. However, there is nothing magical about either 25 or $75 \%$ thumb twitch activity. Although

From the Department of Anaesthesia, University of British Columbia, Vancouver, B.C. the latter indicates nearly as great a degree of recovery as a train-of-four ratio of $0.7^{10}$ which has become the gold standard for adequate neuromuscular activity, "it is still possible to demonstrate clinical weakness until much greater degrees of recovery have been established. ${ }^{12}$ Clinical Duration is usually defined as the time from administration until recovery of adductor pollicis twitch activity to $25 \%$ of control although, again, no studies have determined whether this level of block is appropriate for clinical practice.

In this issue of the Journal, Abdulatif and Taylouni suggest that, given the choice, surgeons may choose a very different level of neuromuscular blockade ${ }^{13}$ from that determined by anaesthetists. In a group of women undergoing Caesarean section with general anaesthesia, neuromuscular blockade was provided with mivacurium administered either by the surgeons in small doses using a syringe pump originally designed for $\mathrm{PCA}$, or by the anaesthetist with an infusion to maintain $95-100 \%$ block of the adductor pollicis. The surgeons used only half as much mivacurium as was given by the anaesthetists, at the end of surgery, spontaneous recovery was much greater after surgical dosing.

It is easy to criticize the study: little abdominal muscle relaxation is required for Caesarean section (but why, then, maintain $95-100 \%$ block?); several factors, such as depth of anaesthesia, may influence the required level of relaxation; and when relaxation is under the control of the operator $\mathrm{s} /$ he may develop the same reassurance and confidence as postoperative patients who may control, but not abolish, their pain by self-administering PCA opioids. However, these results should encourage us to match more closely the level of muscle relaxation with the requirement. Overdosing is wasteful, expensive and may result in postoperative complications. Muscle relaxants are used in several situations in which the required intensity and timing may differ. Rapid and intense, but brief paralysis is needed to facilitate tracheal intubation. Whereas, to provide surgical relaxation, the intensity will depend upon the procedure, supplementary agents and regional anaesthesia but complete recovery should occur rapidly at the end of surgery. Predicting the correct level of paralysis is particularly difficult in the ICU where relaxants are used to enable controlled ventilation. If the goal is paralysis of the relatively resistant respiratory 
muscles, then very large doses will be required, ${ }^{14}$ with the risk of prolonged paralysis. ${ }^{15}$

Abdulatif and Taylouni's report will, no doubt, be followed by others attempting to determine optimal levels of muscle relaxation. Perhaps greater emphasis should be focused, in the early clinical studies of these compounds, on ensuring that the pharmacodynamic behaviour is described in situations which are relevarit to clinical anaesthesia. Drugs should be given in the same combination, doses and sequence as those used in clinical practice rather than to provide the "spurious clinical indices" which often have more to do with the ability to make the measurement than with clinical conditions. After all, who cares about the adductor pollicis, anyway?

Muscle relaxants do not make the hole bigger,

They do not relax bone,

They do not decompress bowel,

They do not give a surgeon judgement, and

They do not relax fat!

(From the Internet, October, 1994)

\section{La profondeur de la curarisation}

Depuis leur introduction en anesthésie, les doses de myorelaxants ont été décidées de façon empirique. Les premières observations portant sur l'usage du "curare " mentionnaient des doses modestes sur fond d'anesthésie profonde au cyclopropane. ${ }^{1}$ Il a fallu attendre 40 ans pour des courbes de relation dose-effet. ${ }^{2}$ On ne donnait pas et on n'avait pas besoin d'antagoniste. Toutefois, avec l'expérience, les doses de myorelaxants ont augmenté et ont atteint un point culminant avec la technique de $\mathrm{Li}$ verpool où, après une généreuse prémédication, on " anesthésiait " au protoxyde d'azote-oxygène en ajoutant de puissantes doses de curare pour la relaxation. ${ }^{3}$ La paix chirurgicale s'obtenait avec un niveau acceptable de curarisation mais on n'avait jamais essayé d'établir si les conditions étaient optimales autant sous l'aspect de la relaxation que sous celui de la récupération fonctionnelle.

Au cours des 20 dernières années, l'évaluation minutieuse d'un grand nombre de nouveaux agents dotés de différences pharmacologiques mineures a permis la création d'une terminologie caractéristique du comportement pharmacodynamique de ces drogues. Par exemple, le délai dinstallation (onset time) est défini comme la mesure du délai situé entre l'adminstration d'un bolus (ou d'une dose importante avec séquence d'amorce) et l'effet maximal observé au niveau de l'adducteur du pouce. Cliniquement, cét intervalle a peu ou pas d'importance maintenant qu'on reconnaît que le délai d'installation de tous les curarisants est plus long au pouce qu'au niveau des muscles respiratoires ${ }^{4}$ et des voies aériennes, ${ }^{5,6}$ qui sont des muscles plus importants parce qu'ils sont susceptibles d'affecter les conditions de l'intubation. Lindex de récupération (recovery index) se définit comme le temps requis pour que le twitch de l'adducteur du pouce récupère de $25 \%$ à $75 \%$ du contrôle.

Instinctivement, on peut déduire qu'une vitesse de récupération rapide diminue la possibilité d'une paralysie résiduelle postopératoire, ce qui, du reste, a déjà été démontré chez les adultes dans plusieurs études. ${ }^{7-9}$ Cependant, il importe peu de constater que l'activité du twitch au pouce soit à $25 \%$ ou à $75 \%$. Bien que la dernière valeur indique un degré de décurarisation comparable au rapport 0,7 sur le train-de-quatre ${ }^{10}$ qui est la règle d'or de la récupération neuromusculaire, ${ }^{11}$ il est toujours possible de démontrer cliniquement la faiblesse jusqu'à ce que des niveaux de récupération plus élevés soient démontrés. ${ }^{12}$ La durée clinique (clinical duration) est ordinairement décrite comme le temps écoulé entre l'administration du relaxant et la récupération du twitch mesuré sur l'adducteur du pouce à $25 \%$ de la valeur du contrôle. Encore là, aucune étude n'a déterminé en clinique la validité de ce niveau de bloc.

Dans ce numéro du Journal, Abdulatif et Taylouni suggèrent que sils avaient le choix, les chirurgiens choisiraient un niveau de curarisation différent ${ }^{13}$ de celui que procurent les anesthésistes. Chez un groupe de parturientes opérées pour une césarienne sous anesthésie générale, la curarisation a été produite avec du mivacurium administré soit par le chirurgien en petits bolus à l'aide d'un pousse-seringue originalement destiné à la PCA, ou par l'anesthésiste grâce à une perfusion suffisante pour maintenir un bloc à $95-100 \%$ de l'adducteur du pouce. Les chirurgiens n'ont utilisé que la moitié de la dose de mivacurium administrée par l'anesthésiste et, à la fin de la chirurgie, la récupération spontanée a été plus rapide après l'administration par le chirurgien.

Ces résultats incitent à la critique: la césarienne requiert peu de relâchement abdominal (pourquoi un bloc à 95-100\%?); plusieurs facteurs, dont la profondeur de l'anesthésie influencent le niveau de relaxation; et lorsque la relaxation est contrôlée par celui ou celle qui opère, il ou elle peut développer la même assurance et la même confiance que le patient qui contrôle sa seringue de PCA mais qui ne supprime pas complètement sa douleur. Cependant, ces résultats devraient nous inciter à adapter 
la relaxation musculaire aux conditions rencontrées. La surdose n'est pas rentable, coûte cher et peut entraîner des complications postopératoires. Une curarisation rapide et intense, mais de courte durée, est nécessaire pour l'intubation de la trachée. Par contre, pour la chirurgie, l'intensité de la relaxation dépend du type d'intervention, des autres agents utilisés et, le cas échéant, de l'anesthésie régionale; il est toutefois essentiel qu'une récupération complète se produise rapidement à la fin de la chirurgie. Prédire le niveau de paralysie est particulièrement difficile à l'unité de soins intensifs où les myorelaxants sont utilisés pour permettre de contrôler la ventilation. $\mathrm{Si}$ on veut paralyser les muscles respiratoires qui sont relativement résistants, de fortes doses seront nécessaires ${ }^{14}$ avec le risque de paralysie prolongée qu'elles comportent. ${ }^{15}$

L'observation d'Abdulatif et Taylouni sera sans doute suivie par d'autres qui tenteront de déterminer le niveau optimal de la relaxation musculaire. Peut-être devraiton insister dès le début de l'investigation d'un produit sur la nécessité d'allier la pharmacodynamic aux situations cliniques. Ces drogues devraient être étudiées avec les mêmes associations pharmacologiques, aux mêmes doses et avec la même chronologie qu'en clinique. Il faut éviter de recourir aux indices factices qui sont plus assimilables à la capacité de mesurer qu'à des conditions cliniques. Entre nous, qui se préoccupe de l'adducteur du pouce?

Les relaxants musculaires nagrandissent pas les trous,

Ne relâchent pas les os,

Ne décompriment pas lintestin.

Ne donnent pas plus de jugement au chirurgien, et $\mathrm{Ne}$ relâchent pas la graisse!

(Extrait d'Internet, octobre 1994)

\section{References}

1 Griffith HR, Johnson EJ. The use of curare in general anesthesia. Anesthesiology 1942; 3: 418-20.

2 Smith CE, Donati F, Bevan DR. Dose-response curves for succinylcholine: single versus cumulative techniques. Anesthesiology 1988; 69: 338-42.

3 Gray TC, Halton J. A milestone in anaesthesia? (dtubocurarine chloride). Proc R Soc Med 1946; 39: 400-10.

4 Lebrault C, Chauvin M, Guirmand F, Duvaldestin P. Relative potency of vecuronium on the diaphragm and the adductor pollicis. Br J Anaesth 1989; 63: 389-92.

5 Meistelman C, Plaud B, Donati F. Neuromuscular effects of succinylcholine on the vocal cords and adductor pollicis muscles. Anesth Analg 1991; 73: 278-82.

6 Donati F, Plaud B, Meistelman C. Vecuronium neuromuscular blockade at the adductor pollicis muscles of the larynx and adductor pollicis. Anesthesiology 1991; 74: 833-7.
7 Viby-Mogensen J, Jørgensen BC, Ording $H$. Residual curarization in the recovery room. Anesthesiology 1979; 50: 539-41.

8 Bevan DR, Smith CE, Donati F. Postoperative neuromuscular blockade: a comparison between atracurium, vecuronium, and pancuronium. Anesthesiology 1988; 69: 272-6.

9 Howardy-Hansen P, Rasmussen JA, Jensen BN. Residual curarization in the recovery room: atracurium versus gallamine. Acta Anaesthesiol Scand 1989; 33: 167-9.

10 Lee C. Train-of -4 quantitation of competitive neuromuscular block. Anesth Analg 1975; 54: 649-53.

11 Ali HH, Wilson RS, Savarese JJ, Kitz RJ. The effect of tubocurarine on indirectly elicited train-of-four muscle response and respiratory measurements in humans. $\mathrm{Br} \mathrm{J}$ Anaesth 47: 570-4, 1974.

12 Stanec A, Nuesa W, Akturk A, Pillon, Capek $K$. Recovery of respiratory muscle function in surgical outpatients. Anesthesiology 1990; 73: A878.

13 Abdulatif $M$, Taylouni $E$. Surgeon-controlled mivacurium administration during elective Caesarean section. Can J Anaesth 1995; 42: 96-102.

14 Chauvin M, Lebrault C, Duvaldestin P. The neuromuscular blocking effect of vecuronium on the human diaphragm. Anesth Analg 1987; 66: 117-22.

15 Gooch JL, Suchyta MR, Balbierz JM, et al. Prolonged paralysis after treatment with neuromuscular blocking drugs. Crit Care Med 1991; 19: 1125-31. 